\title{
Spread of Hemagglutinating Encephalomyelitis Virus (HEV) in the CNS of Rats Inoculated by Intranasal Route
}

\author{
${ }^{1}$ NORIO HIRANO, ${ }^{3,4}$ KOUJIRO TOHYAMA, ${ }^{2}$ HIDEHARU TAIRA, AND \\ ${ }^{4}$ TSUTOMU HASHIKAWA \\ ${ }^{I}$ Department of Veterinary Microbiology, and ${ }^{2}$ Department of Bioscience and Technology, \\ Iwate University, Morioka 020-8550; ${ }^{3}$ The Center for Electron Microscopy and Bio-imaging \\ Research and Department of Neuroanatomy, Iwate Medical University, Morioka 020-8505; \\ ${ }^{4}$ Laboratory of Neural Architecture, Brain Science Institute, RIKEN, Wako 351-0198, Japan
}

\section{INTRODUCTION}

HEV is known to cause vomiting and wasting disease or encephalomyelitis in piglets (Andries et al., 1980,1981; Mengeling et al., 1972; Roe et al., 1958). In experimental oronasal infection of piglets, the virus spread to the CNS predominantly via the nerve pathways (Andries et al., 1980, 1981). In our previous study, 4-week-old rats died of encephalitis after inoculation by different routes including intranasal route (Hirano et al., 1993). In the infected animals, the virus reaches the CNS through the nerve pathways from peripheral nerve. Our previous studies have demonstrated that HEV propagated through nervous route and its infection was restricted to neurons after inoculation into sciatic nerve or footpad of rats (Hirano et al., 1995, 1998). Our findings suggest that HEV is useful as a trans-synaptic tracer for analyzing neuronal connections in the CNS.

In the present study, we attempt to examine and evaluate the usefulness of HEV as a trans-synaptic tracer in the olfactory pathways. In addition, we examine the possibility whether this system provides a model for analysing the natural infection in pigs.

The Nidoviruses (Coronaviruses and Arteriviruses).

Edited by Ehud Lavi et al., Kluwer Academic/Plenum Publishers, 2001. 


\section{MATERIALS AND METHODS}

Plaque-purified HEV 67N strain (Mengeling et al., 1972) was propagated and assayed for infectivity in SK-K cells as described previously (Hirano et al., 1990). SPF of 4- to 6-week-old Wistar male rats were used. Twenty $\mu$ l of the virus $\left(1 \times 10^{5} \mathrm{PFU}\right)$ was inoculated into the right nasal cavity of rats. On days 1 to 7 after inoculation, rats were killed under deep anesthesia with pentobarbital and the brain and spinal cord were dissected out and assayed. For immunostaining, rats were perfused with $4 \%$ paraformaldehyde in $0.1 \mathrm{M}$ phosphate buffer under deep anesthesia on day 3 to 13 . The brain was dissected out and sections were obtained on a freezing microtome, treated with anti-HEV $67 \mathrm{~N}$ mouse antibody $(1: 1000)$ at $4 \mathrm{C}$ overnight, and reacted with FITC-conjugated goat serum (anti-mouse $\mathrm{IgG}$ ) at room temperature for 2 hours. The stained sections were examined under confocal laser scanning microscope.

\section{RESULTS}

Inoculated rats became hypersensitive on day 7 to 10 and began to die showing CNS signs on day 10 . On day 2, HEV was first isolated from the ipsilateral brain but not from the contralateral. On day 3, the virus was isolated from the contralateral brain and its titer was about one tenth of the ipsilateral brain. On day 4, the virus was detected from the spinal cord. Until day 5 to 7 , the infectivity titers of the contralateral side reached nearly the same level as in the ipsilateral brain, $10^{6}$ to $10^{7} \mathrm{PFU} / 0.2 \mathrm{~g}$. HEV antigen was found in the olfactory bulb ipsilateral to the inoculated nasal cavity on day 3 . On day 4 in main olfactory bulb (MOB), many positive neurons located in mitral cell and external flexiform layers (Fig.1a). Many immuno-positive neurons were found in anterior olfactory nucleus (AON) (Fig.lb) and in the olfactory cortex (OC) (Fig.1c). Day 6, additional area including septal (SNT) and paraventricular nuclei (PVT) of thalamus contained many antigen positive neurons (Fig. 2a). Neurons in the superior colliculus (SC) and a few Purkinje and granular cells in the cerebellar cortex (CC) exhibited antigenpositive (data not shown). Day 13, pyramidal cells in hippocampus (CA) and granular cells in dentate gyrus (DG) became infected (Fig.2b), which differ from the findings on day 6 (Fig.2a). In OC, the number of antigen positive neurons did not increase. Neurons appeared to be antigen positive in the inferior colliculus (IC) where no immunostained neurons were found on day 6 . The antigen was detected predominantly in neurons but not in glial cells. No cytopathological evidence was found in HEV infected neurons. 


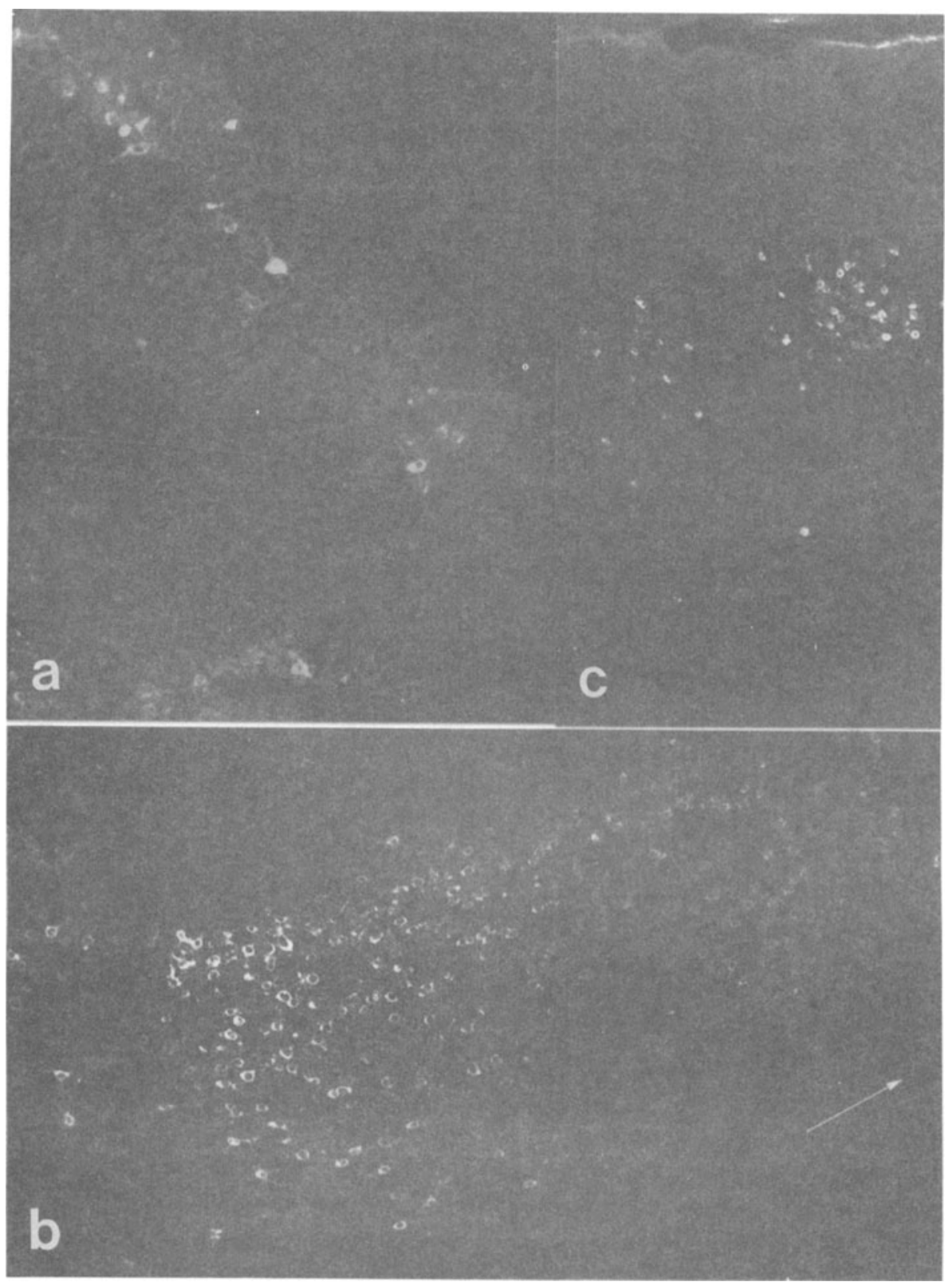

Figure 1. On day 4, a) HEV positive neurons including mitral cells in ipsilateral olfactory bulb; b) anterior olfactory nucleus containing numerous positive neurons; c) primary olfactory cortex. 


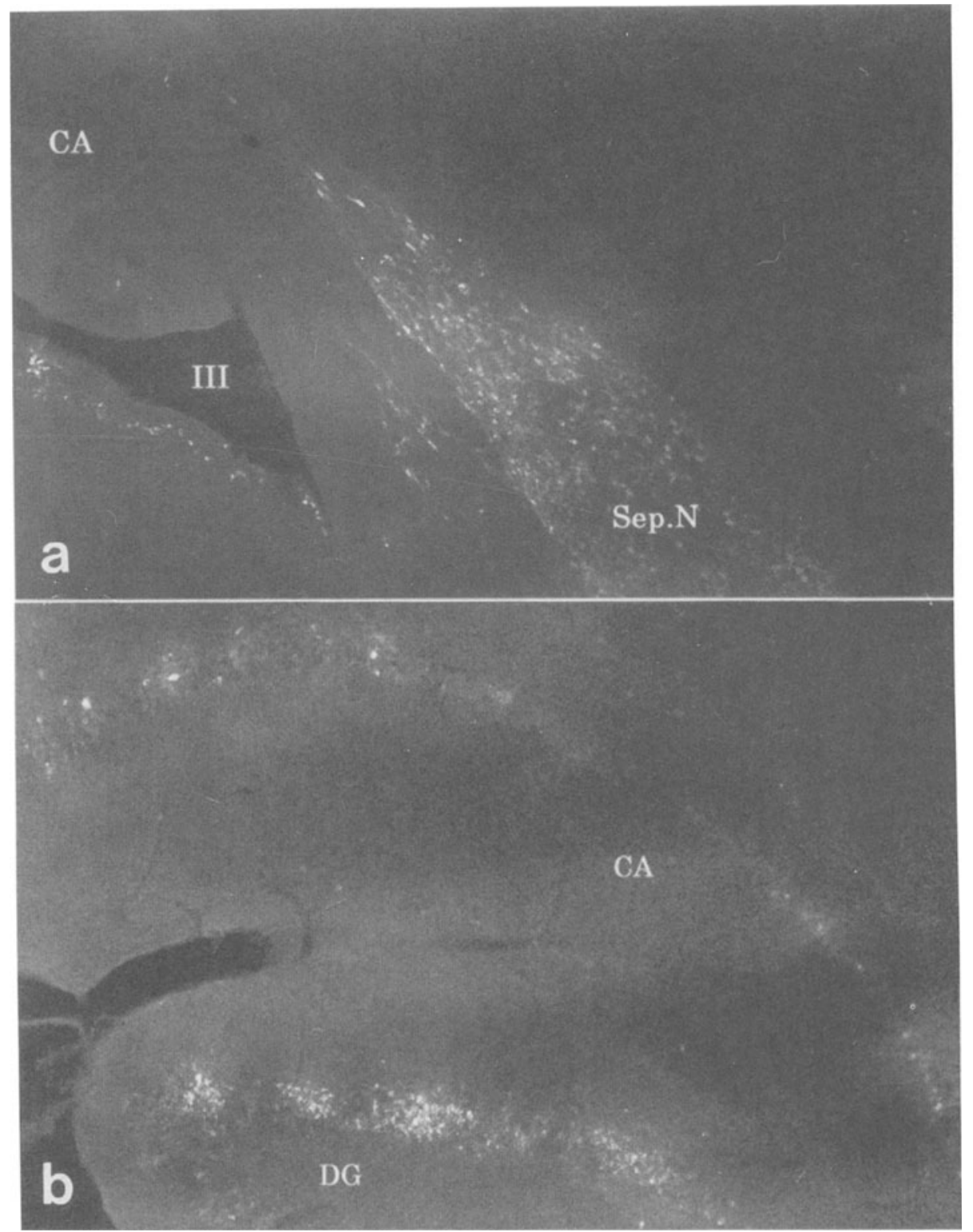

Figure 2. On day 6 (a) and 13(b), a) antigen positive neurons in the septal nucleus (Sep.N) and in paraventricular thalamic nucleus but not in the hippocampus (CA), III: 3rd ventricle; b) antigen positive pyramidal cells in the hippocampus (CA) and granular cells in dentate gyrus (DG). 


\section{DISCUSSION}

In rats, two components of the olfactory system are recognised: the main and accessory olfactory systems. In the present study, HEV positive neurons distributed in MOB, AON, OC, SNT, PVT, SC, CC, CA, DG, and IC. MOB and $\mathrm{OC}$ are well known structures of the main olfactory pathway. MOB, AON, and some limbic nuclei including SNT, PVT, CA and DG belong to accessory olfactory system. Positive neurons in other components (SC, CC and IC) may infect through trigeminal nerve route.

Andrires and Pensaert (1980) reported the trigeminal nerve route as well as olfactory pathway in piglets after oronasal inoculation of HEV. Lavi et al. (1988) described the virus spread through the limbic system in mice after intranasal inoculation of mouse hepatitis virus (MHV). Our observations in the present study are coincident with the results of these reports. HEV antigen was detected predominantly in neurons, but not in glial cells, of olfactory pathways as well as trigeminal route in the present study. This result indicates that HEV is confirmed to be extremely neurotropic virus different from others including MHV and herpes simplex virus, which infects not only neurons but also surrounding glial cells (Lavi et al., 1988; Cook et al., 1973). In other word, HEV is a causative agent, which has strict neurotropism. In addition, no distinct degenerating changes were found in HEV infected neurons, suggesting that the fiber connections along olfactory pathways and other related fiber connections kept intact as far as the period examined. In general, such properties of HEV have the advantage of analyzing neuroanatomical connections as a trans-synaptic tracer. In the olfactory pathways, however, some other related fiber connections make it difficult to investigate anatomical connections using HEV. Still, it retains the possibility that more precise study may resolve this complication and reveal the systems combined with other tracers.

\section{ACKNOWLEDGMENTS}

This study was supported by the Grands-in-aid (No. 11660309) from the Ministry of Education and Culture of Japan.

\section{REFERENCES}

Andries, K., and Pensaert, M.B. 1980. Immunofluorescence studies on the pathogenesis of hemagglutinating encephalomyelitis virus infection in pigs after oronasal inoculation. Am. J. Vet. Res. 41: 1372-1385. 
Andries, K., and Pensaert, M.B. 1981. Vomiting and wasting disease; a coronavirus infection of pigs. Adv. Exp. Med. Biol. 142: 399-408.

Hirano, N., Haga, S., and Fujiwara, K. 1993. The route of transmission of hemagglutinating encephalomyelitis virus (HEV) $67 \mathrm{~N}$ strain in 4-week-old rats. Adv. Exp. Med. Biol. 342: 333-338.

Hirano, N., Nomura, R., Tawara, K., Ono, K., and Iwasaki, Y. 1995. Neuronal spread of hemagglutinating encephalomyelitis virus (HEV) $67 \mathrm{~N}$ strain in 4- week-old rats. $A d v$. Exp. Med. Biol. 390: 117-119.

Hirano, N., Ono, K., Takasawa, H., and Haga, S. 1990. Replicaiton and plaque formation of swine hemagglutinating encephalomyelitis virus $(67 \mathrm{~N})$ in swine cell line, SK-K culture. $J$. Virol. Methods 27:91-100.

Hirano, N., Tohyama, K., and Taira, H. 1998. Spread of hemagglutinating encephalomyelitis virus from peripheral nerve to the CNS. Adv. Exp. Med. Biol. 440: 601-607.

Lavi, E., Fishman, P., Highkin, M., and Weiss, S. 1988. Limbic encephalitis after inhalation of a murine coronavirus. Lab. Invest. 98: 31-36.

Mengeling, W.L., Boothe, A.D., and Richite, A.E. 1972.Characterization of a coronavirus (Strain 67N) of pigs. Am. J. Vet. Res. 33: 297-308.

Mengeling, W.L., and Cutlip, R.C. 1976. Pathogenicity of field isolates of hemagglutinating encephalomyelitis virus for neonatal pigs. J. Am. Vet. Assoc. 68: 236-239.

Roe, C.K., and Alexander, T.J.L. 1958. A disease of nursing pigs previously unreported in Ontario. Canad. Comp. Med. 22: 305-307. 